Thorax (1966), 21, 193.

\title{
Pedunculated haemangiopericytoma attached to the thoracic aorta
}

\author{
W. K. BLENKINSOPP AND J. T. HOBBS \\ From the Department of Pathology and the Surgical Unit, St. Mary's Hospital, London W.2
}

In 1942 Murray and Stout reported on tissue culture of the 'epithelioid' cells of the glomus tumour and suggested that they were the pericytes of Zimmermann. In the same year these authors (Stout and Murray, 1942) published the first description of another tumour of the same cells which they named 'haemangiopericytoma'. Since then more than 300 examples of this tumour have been reported, at least $90 \%$ of them in America. This paper describes an unusual presentation of haemangiopericytoma and remarks on the rarity of reported cases in this country.

\section{CASE REPORT}

In 1959 a woman of 63 noticed a 'scratching' sensation in her chest, but a chest radiograph at the Hampstead Chest Clinic showed no abnormality. Five years later she had acute bronchitis; this quickly cleared up with a course of tetracycline, but a chest radiograph at this time showed a mass in the paraaortic region. Subsequent radiographic examinations showed that the mass was increasing in size, and in January 1965 the patient was referred to the thoracic surgical unit at St. Mary's Hospital with a tentative diagnosis of aortic aneurysm.

The patient gave a history of winter cough with sputum for six years; she had noticed that left chest discomfort was brought on by coughing and deep inspiration, and sometimes it was relieved by choline theophyllinate. Her weight was increasing. Examination showed a fit, slightly overweight, elderly woman with no cardiovascular abnormalities except a blood pressure of $150 / 100 \mathrm{~mm} . \mathrm{Hg}$; the pulse was regular at $73 /$ minute. The chest was emphysematous, and there were scattered rhonchi in both lung fields. The abdomen was obese, but no viscus or abnormal mass was palpable.

INVESTIGATIONS Haemoglobin $99 \%$, packed cell volume $45 \%$, white cell count $7,000 /$ c.mm.; sedimentation rate $16 \mathrm{~mm}$. $/ \mathrm{hr}$.; plasma electrolytes normal, plasma urea $30 \mathrm{mg}$. $/ 100 \mathrm{ml}$., serum calcium $10 \mathrm{mg} . / 100 \mathrm{ml}$. ;
Wassermann reaction and Harris test both negative ; blood group $\mathbf{O}$ rhesus-positive; electrocardiogram normal. The forced vital capacity was $1,130 \mathrm{ml}$., and the ratio of forced expiratory volume in one second to the forced vital capacity was $63 \cdot 5 \%$. Chest radiographs (Fig. 1) revealed a large rounded mass in the left paravertebral gutter, and chest tomography showed normal thoracic vertebrae with the main mass posterior to the aortic plane but extending to the aortic arch; an arch aortogram via the left axillary artery showed a normal lumen and no filling of the mass.

A diagnosis of aortic aneurysm was thus not confirmed by angiography, and a provisional diagnosis of neurofibroma was doubted because there was no extension towards the intervertebral foramina and no widening of the rib spaces locally ; the possibility of an enteric cyst was considered. Because the mass had been rapidly increasing in size a surgical exploration was decided upon, and on 9 March 1965 the left chest was opened through the seventh intercostal space. There was no pleural effusion and the pleura and left lung appeared normal, but when the lung was displaced forwards a tense, fleshy, mobile tumour, about $8 \mathrm{~cm}$. long, was seen in the paravertebral gutter, attached by a pedicle to the anterolateral aspect of the descending aorta at the level of T8; there was no local infiltration. The mass was easily removed when a cleavage plane was formed in the outermost layers of the adventitia of the aorta, and the left thoracotomy was then closed. The patient's recovery was uneventful; and three months later she was symptom-free.

HISTOLOGY The specimen was a firm, encapsulated mass, measuring 8.5 by 5.0 by $4.5 \mathrm{~cm}$., including at one end a pedicle $1.5 \mathrm{~cm}$. long and $3 \mathrm{~cm}$. in diameter. The cut surface of the tumour was fleshy, and the base of the pedicle was composed of loose connective tissue. Histologically the tumour consisted of very numerous capillaries separated by fairly regular rounded cells (Figs 2 and 3). Reticulin staining showed the cells to be lying outside the capillary sheaths, and most of the cells were surrounded by reticulin fibres; the reticulin was not arranged in alveoli. Scattered among the tumour cells there were 


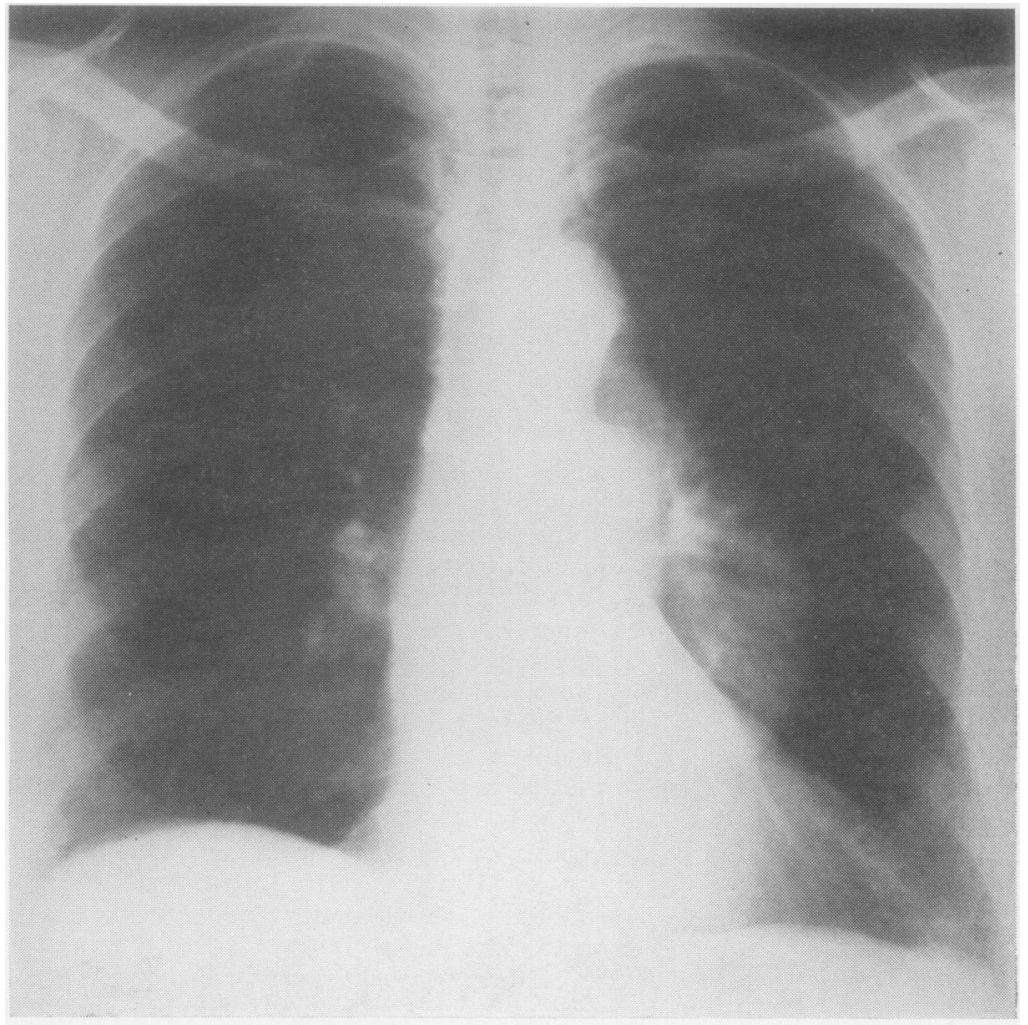

FIG. 1. Chest radiographs, (a) postero-anterior, and (b) left. lateral, taken in February 19650 show a mass in the left paraver tebral gutter.

(a)

mast cells and a few plasma cells. There were very few mitoses. Nerve fibres were not found. Thus the tumour showed the features typical of haemangiopericytoma. There were three unusual features: some areas had a fibromatous appearance-spindle cells intimately associated with collagen (Fig. 4); in the pedicle the capillary walls were thickened by collagen; pedunculation is extremely rare in a haemangiopericytoma.

\section{DISCUSSION}

Blood can pass from arteriole to venule either through a capillary network or through a shortcircuiting vessel; this short-circuiting vessel is enveloped by cells known as Zimmermann's pericytes, which at either end of the vessel merge morphologically into smooth muscle cells; pericytes have been found all over the body and presumably occur wherever capillary networks exist.

Stout (1956) discussed the evidence that haemangiopericytoma arises from these cells, and 
several authors have described the appearance and behaviour of the tumour. It is surprising that so few have been reported in this country, and, in view of this apparent reluctance to make the diagnosis, sections of the present case were sent to Professor A. Purdy Stout in New York, and to Professor I. Doniach, Professor R. Willis, and Dr. R. W. Evans in this country, each of whom considered the tumour to be a haemangiopericytoma.

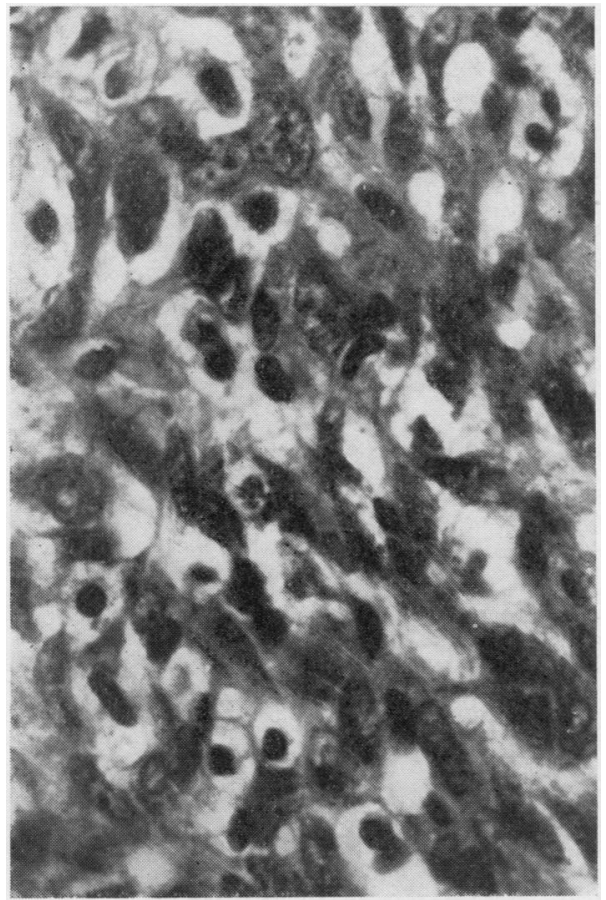

FIG. 2. Haemangiopericytoma, showing the large tumour cells with vesicular nuclei and scattered mast cells and plasma cells. Many of the capillaries are partly collapsed and can be identified only at higher magnification $(H$. and E., $\times 672$ ).

About two-thirds of the haemangiopericytomata reported by Stout (1949) and Fisher (1960) occurred in the superficial soft tissues of the limb, although O'Brien and Brasfield (1965) found nine in the trunk and seven in the head among 24 collected cases. In our patient the tumour was attached to the descending thoracic aorta and probably arose in the connective tissue between the aorta and the pleura.

The benign and malignant forms are difficult or impossible to distinguish histologically. Stout (1956) described the occurrence of metastases in $11.7 \%$ of 197 cases, but commented: 'There has

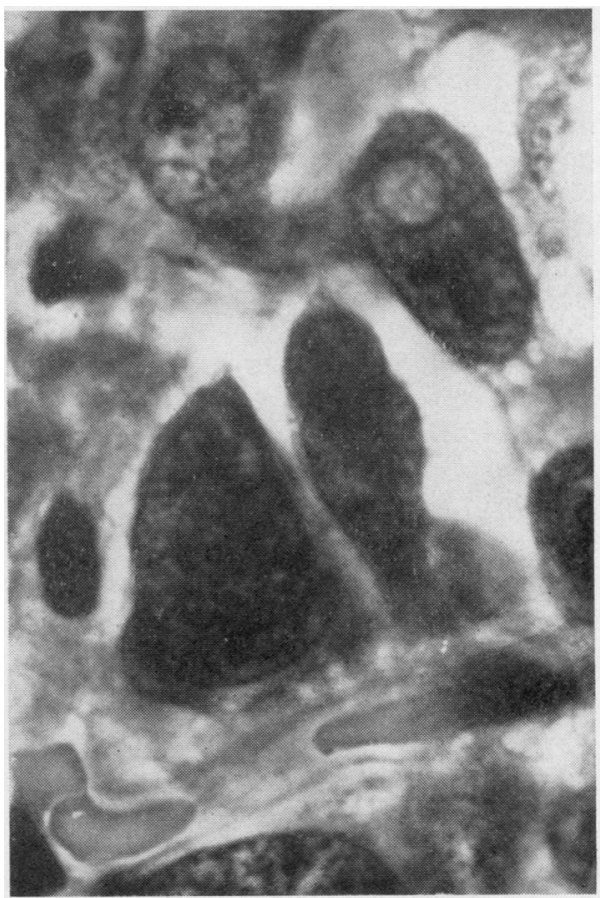

FIG. 3. Haemangiopericytoma cells intimately related to a capillary $(H$. and $E ., \times 1,680)$.

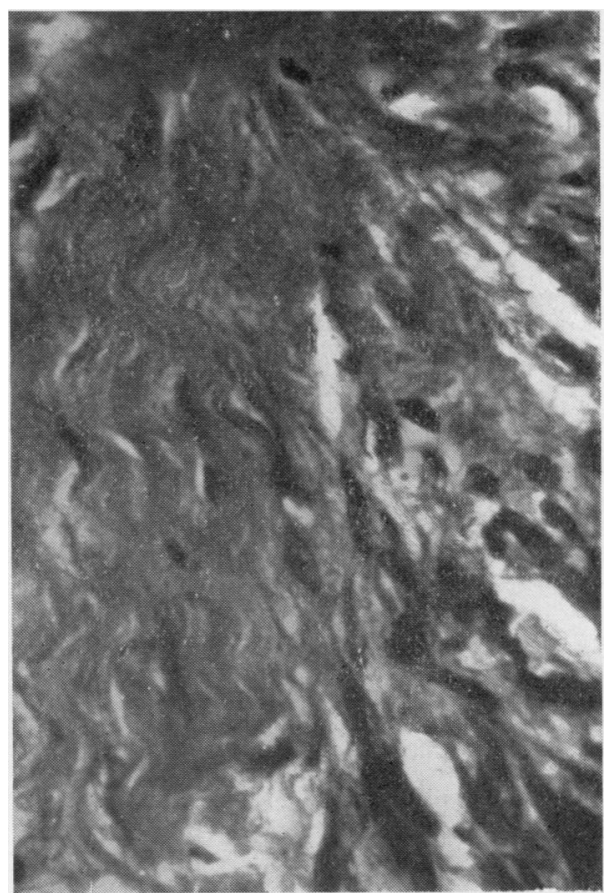

FIG.4. Fibromatous area of the tumour (H.and E., $\times 672$. 
not been time to obtain accurate follow-up data on the patients with these tumours.' More recently, Fisher (1960) found metastases in nine of 20 cases $(45 \%)$; the tumour recurred locally in eight of the cases with metastases and in four without metastases. O'Brien and Brasfield (1965) found metastases in 13 of 23 cases, the sites being lung (13), bone (4), liver (8), and lymph node (3).

The pathological differential diagnosis includes haemangioendothelioma, chemodectoma, and glomus tumour. In haemangioendothelioma the proliferated cells lie inside the reticulin sheath of the capillaries. In chemodectoma the cells are arranged in definite alveoli, each nest of cells being enclosed by reticulin. The glomus organ includes pericytes, but occurs almost exclusively in the subcutaneous tissue, and the glomus tumour has an organoid appearance and many nerve fibres and is painful.

Collagenous thickening of the capillaries, seen in the present case, has been reported in three cases by Stout (1949), but this has also been found in chemodectoma (Barrie, 1961) and therefore is not of diagnostic assistance. The production of fibromatous tissue in our case is an indication of the close relation between various connective tissue cells. Indeed, haemangiopericytoma resembles the highly cellular fibroma, often called fibrosarcoma of low-grade malignancy, which behaves in much the same manner.

\section{SUMMARY}

Haemangiopericytoma is an uncommon tumour. It is rarely pedunculated, and its origin from the thoracic aorta has not hitherto been reported. A patient with an unusual presentation of such a tumour is described. Because of an apparent reluctance to diagnose haemangiopericytoma in this country the pathological features are discussed.

We are grateful to Dr. Shneerson, of the Hampstead Chest Clinic, who referred the patient; to Professors I. Doniach, R. Willis, and A. Purdy Stout, and Dr. R. W. Evans, who kindly gave their opinions on sections from this tumour; and to Mr. L. L. Bromley, director of the thoracic surgical unit at St. Mary's Hospital, under whose care the patient was admitted.

\section{REFERENCES}

Barrie, J. D. (1961). Intrathoracic tumours of carotid body type (chemodectoma). Thorax, 16, 78.

Fisher, J. H. (1960). Hemangiopericytoma: A review of twenty cases. Canad. med. Ass. J., 83, 1136.

Murray, M. R., and Stout, A. P. (1942). The glomus tumor: investigation of its distribution and behavior, and the identity of its 'epithelioid' cell. Amer. J. Path., 18, 183.

O'Brien, P., and Brasfield, R. D. (1965). Hemangiopericytoma Cancer (Philad.), 18, 249.

Stout, A. P. (1949). Hemangiopericytoma: A study of twenty-five new cases. Cancer, 2, 1027.

(1956). Tumors featuring pericytes: Glomus tumor and hemangiopericytoma. Lab. Invest., 5, 217.

and Murray, M. R. (1912). Hzmangiopericytoma: a vascular tumor featuring Zimmermann's pericytes. Ann. Surg., 116, 26. 